\title{
Midface Reconstruction
}

\author{
Ömer Özkan, M.D., ${ }^{1}$ O. Koray Coşkunfirat, M.D., ${ }^{1}$ and Özlenen Özkan, M.D. ${ }^{1}$
}

\begin{abstract}
In the midface, two polyhedron-shaped maxillary units are separated by the central midportion, which includes the nasal area. The midface includes such facial features as the nose, cheek, and upper lip, and posteriorly it extends to the anterior skull base. In the superoinferior direction, the midface includes the soft and bony tissue from the orbital cavity to the oral cavity. Laterally, the midface extends to the temporal bone. Although most superficial skin defects of the midface can be covered by various standard reconstructive modalities, because of the need to evaluate the nature of the tissues involved and because of structural and also functional considerations, the management of large, fullthickness defects is a challenge for reconstructive surgeons. Advances in microsurgical techniques have permitted reliable wound closure and a substantial decrease in patient morbidity with low complication rates while allowing a variety of reconstructive flap options in a single stage. To create a reconstructive algorithm, several classification systems have been proposed, mostly relating to the extension, location, and tissue involvement of the defect. Defects can be classified as simple soft tissue defects and complex defects. The complex three-dimensional defect is classified under four types: types I to IV. Although maxillary prostheses are nonliving tissues and may cause discomfort for the patient, in special situations they can be reconstructive options requiring special experience. Essentially, the method of reconstruction should be selected on an individual basis, bearing in mind the medical situation; the age and prognosis of the patient; the size, extension, and composition of the defect; and the availability of local or distant tissues.
\end{abstract}

KEYWORDS: Midface

The midface includes the most prominent features of the face and separates the oral cavity from the orbital cavity. It resembles a polyhedron-shaped box maintained by a bony framework. In addition to its structural anatomic properties, the midface has functional properties with regard to respiration, speech, mastication, swallowing, vision, and olfaction. In the midface, two polyhedron-shaped maxillary units are separated by the central midportion, which includes the nasal area. The midface includes such facial features as the nose, cheek, and upper lip, and posteriorly it extends to the anterior skull base. In the superoinferior direction, the midface includes the soft and bony tissue from the orbital cavity to the oral cavity. Laterally, the midface extends to the temporal bone. All these structures include various tissues; the main bony architecture is surrounded by muscles, skin, and mucosa that have specific relations with a harmonious interconnection.

In the upper portion, the midface has a supportive function for the orbital contents; inferiorly, it constitutes the ceiling of the oral cavity. In the middle portion, the midface also separates the airway and olfactory units

\footnotetext{
${ }^{1}$ Department of Plastic and Reconstructive Surgery, Akdeniz University Hospital, Antalya, Turkey.

Address for correspondence and reprint requests: Ömer Özkan, M.D., Akdeniz Üniversitesi Hastanesi, Plastik ve Rekonstrüktif Cerrahi Anabilim Dali, B Blok kat 2, 07059, Antalya, Turkey (e-mail: omozkan@hotmail.com).

Advances in Head and Neck Reconstruction, Part I; Guest Editors,
}

Samir Mardini, M.D., Christopher J. Salgado, M.D., and Hung-Chi Chen, M.D., F.A.C.S.

Semin Plast Surg 2010;24:181-187. Copyright (C) 2010 by Thieme Medical Publishers, Inc., 333 Seventh Avenue, New York, NY 10001, USA. Tel: +1(212) 584-4662.

DOI: http://dx.doi.org/10.1055/s-0030-1255335.

ISSN 1535-2188. 
with its complex bony framework. The midface resembles a box full of air. The air-filled sinuses have an important impact on respiration aerolization, and also reduce unnecessary weight in the midface. These sinuses are valuable in maintaining the anterior volume of the midface to protect the vitally important brain, thus reducing the degree of anterior impaction forces. The main supportive structures, the so-called buttresses of the bones, hold the midface in its normal anatomic position. These buttresses are mainly divided into two types: vertical and horizontal. The vertical buttresses are nasofrontal, pterygomaxillary, and zygomaticomaxillary. These three buttresses provide the midface with the resistance to be able to maintain its upright position. The vertically oriented buttress in the direction of the maxillary alveolus forms the upper jaw and performs a supportive function for the teeth.

The muscles controlling facial expression are located in the midface and covered by the facial skin. The outer surface of the face is divided into the cheek, nose, and lip. All these units have different characteristics and call for special attention in reconstruction. The parotid gland is another important structure in the midface and is located in the most lateral portion. This is not only a salivary gland but also forms a soft tissue bulk for the facial contour.

The arterial blood supply comes mainly from the branches of the external carotid artery. Although the facial artery plays the most significant role in supplying the cheek, there are several anastomoses between the branches of the external carotid artery and other arteries, which makes for a rich vascular network, especially in the skin (e.g., infraorbital and terminal branches of the facial artery, and branches of the internal maxillary artery). This rich anastomotic branching pattern is particularly important during resectional surgery when sacrificing some of the major arteries to leave no impairment to the vascular supply of the remaining structures. However, it should be kept in mind that this abundant blood supply may create a significant difficulty in providing hemostasis after major resections. The functional and sensorial innervations of the midface structures are mainly provided by two cranial nerves. Whereas the mimetic muscles of the midface are innervated by the facial nerve (cranial nerve VII), the masticatory muscles are innervated by the trigeminal nerve (cranial nerve V). Sensory innervation is supplied by the branches of the trigeminal nerve. The lymphatic pathways drain the midface toward the parotid and submaxillary lymph nodes. ${ }^{1-3}$

\section{TYPES OF DEFECT}

Midfacial defects may occur in several ways, including iatrogenic surgical factors due to tumor resections, trauma, congenital anomalies, and inflammatory and degenerative disorders. The severity of a defect is concordant with the extent of tissue loss, the relation between the defect and adjacent structures and cavities such as the brain and oral cavity, and involvement of vital structures such as orbital contents and nerves (Fig. 1).

Whatever the reason for tissue loss, the ultimate goal in reconstruction should include the provision of a three-dimensional replacement for the missing parts with the best available similar tissue, the provision of an acceptable cosmetic appearance, and the maintenance of the functional status of the regional anatomy, such as separating the oral cavities from the upper neighboring structure, providing a respiratory tract, and supporting the orbital contents in suitable cases.

\section{Soft Tissue Defects}

Soft tissue defects of the midface include those of the cheek, nose, upper lip, and underlying muscles. There may be several reasons for such defects, including trauma, burns, tumor resection, Romberg's disease, infection, radiotherapy, congenital deformities, and vascular malformations. Most soft tissue defects are not complicated and can be managed using skin grafts or local flaps. Because skin grafts carry the risk of inevitable contraction, a shiny appearance, and pigment changes, the use of skin grafts is usually limited. If it is decided to use a skin graft, the full-thickness type is to be preferred as it contracts less and carries less risk of pigment changes. The best options for full-thickness skin graft sources are postauricular, preauricular, and supraclavicular regions, depending on the thickness of the defect and mismatch between the color of the defect and that of the donor area. If a larger skin graft is needed, the inguinal region can also be selected. For small defects for which primary skin closure or skin grafting is undesirable or not suitable, several types of local flap can be fashioned. Rotation, transposition, or advancement flaps can be used.

Although for external nasal coverage, rotation and advancement flaps are mostly used, the rhomboid flap is the main local flap of choice for the cheek region. Although historically it has been of paramount importance for large nasal defects, the paramedian forehead flap is still commonly used in clinical practice. Although it has a relatively high complication rate in this region, the tissue expansion technique is a good alternative to resurface the defect with an analogous color and texture, especially for resectional surgery due to scar revision or large nevi. Sometimes the lack of soft tissue can be great, as in Romberg's disease or tumor resection that can best be treated by the transfer of a large amount of soft tissue. In such cases, free tissue transfer is the best alternative to make good the soft tissue deficiency.

For coverage of the external skin and/or underlying soft tissue, several flaps can be selected. Radial forearm, anterolateral thigh (ALT), or scapular flaps are 
A
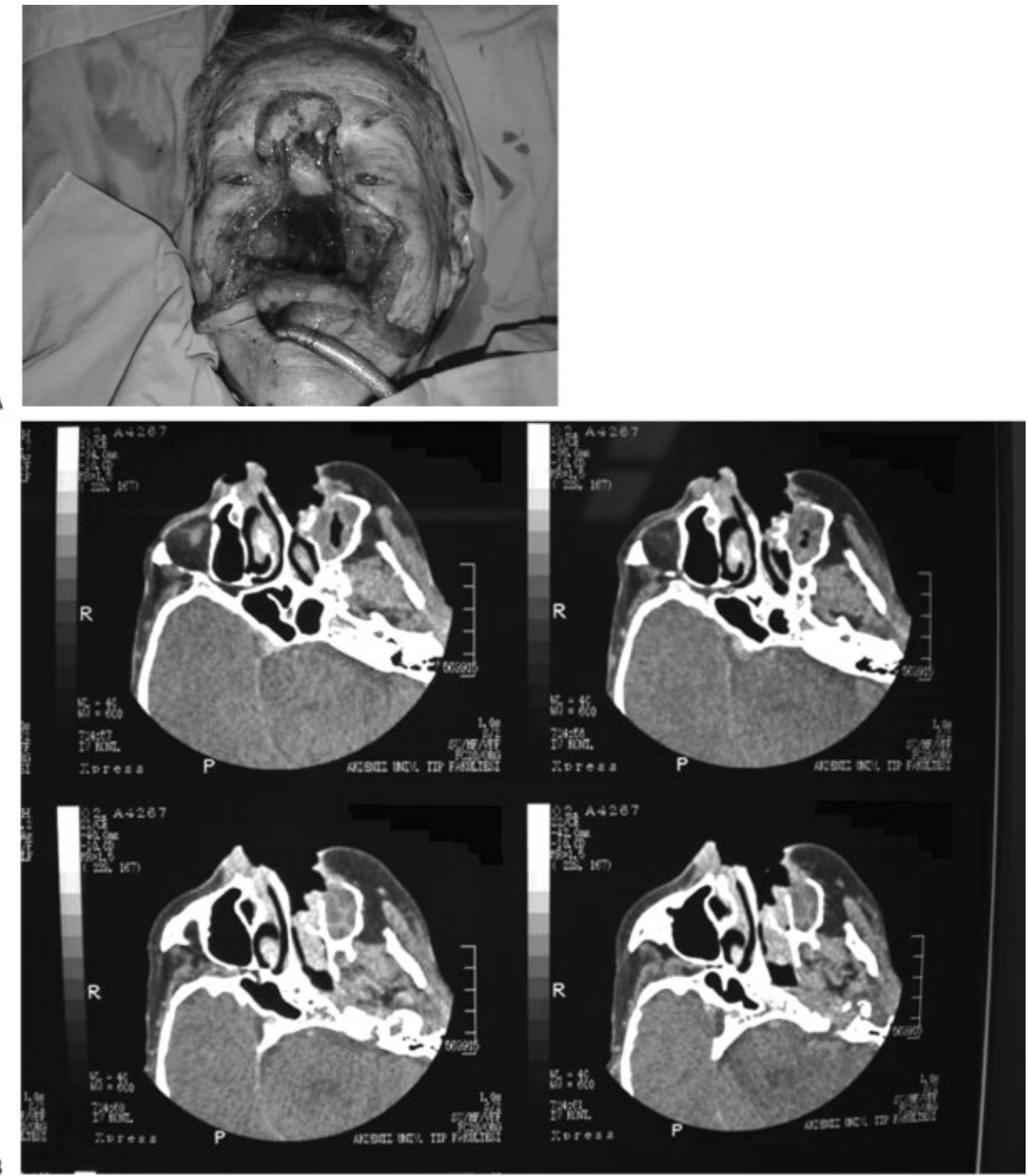

Figure 1 (A) Extensive midface defect with exposure of cranial base after resection of a squamous cell carcinoma. (B) Preoperative computed tomographic view of the patient.

the best choices for external coverage, although the scapular flap is the least attractive alternative because of the need for a position change during surgery. The radial forearm flap is a good choice because it is very thin and reliable, although the donor site scar is unsightly, and one of the major arteries of the hand has to be sacrificed (Fig. 2). The ALT flap is very popular because its vascular pedicle is sufficiently long and large, it can be thinned safely, and the donor area scar can be easily concealed. The dorsalis pedis flap is a good alternative for large nasal defects in terms of its pliable and thin skin nature; however; it has a considerable donor site morbidity (Fig. 3).

When the soft tissue defect is more prominent, bulkier flaps are selected, such as rectus abdominis, latissimus dorsi, and ALT myocutaneous flaps. Again, the ALT flap seems the most favorable choice because of its lower donor site morbidity, adjustable flap thickness, and availability for a two-team approach.

For free flap transfers to the midface, facial vessels and superficial temporal vessels are mostly selected; however, in some situations such as large tumor excisions, neck vessels (e.g., superior thyroid, external carotid artery) may be the best choice, and flaps with longer pedicles such as the anterolateral flap, latissimus dorsi, or rectus abdominis are selected. When soft tissue restoration is achieved using bulky flaps, secondary flap revision is usually necessary to obtain a better contour and cosmetic appearance.

Sometimes, soft tissue deficiency or the resection involves the facial muscles and nerves, resulting in a loss of facial expression. In such patients, facial reanimation is necessary in addition to contour restoration. The restoration technique depends on the type of defect; if 


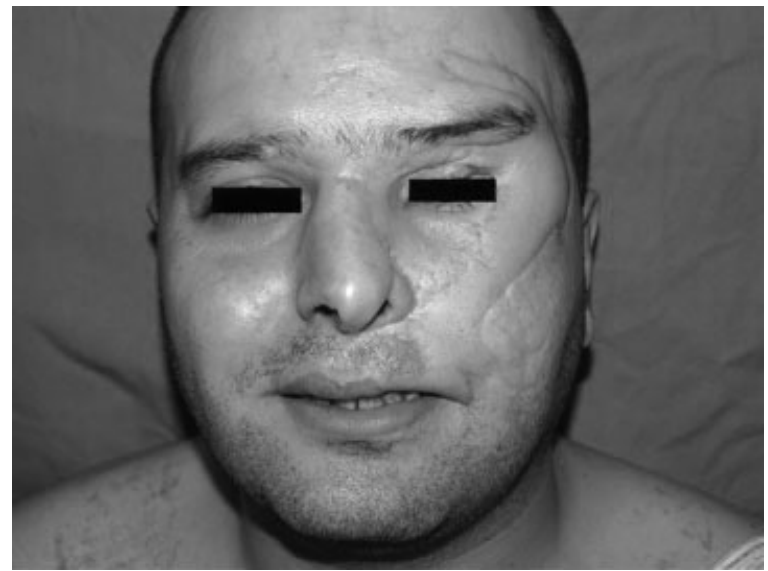

Figure 2 Soft tissue reconstruction of large complex upper lip, cheek, eyelid, and forehead defects with radial forearm flap. Postoperative view at 24 months.

only the nerve is involved, nerve grafts can be used. Nevertheless, functional muscle transfer is necessary because of long-lasting paralysis or damaged facial muscles. For functional muscle transfer, local muscles can be transferred, such as the temporal and masseter muscles. The limitations of local muscles have encouraged surgeons to use free muscle transfers because more powerful muscle units can be transferred and oriented in the correct direction. These transferred muscles may be innervated by the remaining or contralateral facial nerve so that symmetrical contractions may be possible. The gracilis, latissimus dorsi, rectus abdominis, serratus anterior, and pectoralis minor muscles are the alternatives for facial reanimation.

\section{Complex, Three-Dimensional Defects}

This type of defect involves the bony framework (bony and cartilaginous for the nose), meaning that the structure of the midface has collapsed. Skeletal defects may be accompanied by external soft tissue and/or internal mucosal defect. This kind of defect is also related to the aerodigestive system. The maxillary bone can be divided into three segments for a better understanding of threedimensional defects and affected structures. These segments are superior, middle, and lower. The lower third separates the nasal cavity and the maxillary sinus from the oral cavity. This part constitutes the palatal ceiling and serves as a bone stock for dentition. The lower part is also supportive of the midface as the horizontal buttress. The middle part forms the outer walls of the maxillary sinus and the lateral wall of the nasal cavity. The middle part includes a large chamber filled with air, the lateral walls of which are the two important vertical buttresses (nasomaxillary and zygomaticomaxillary) of the midfacial skeleton. The upper portion separates the maxillary sinus from the orbital cavity and also serves as the upper lateral boundary of the nasal cavity and separates it from the orbital cavity. The malar eminence provides the prominent appearance of the midface. This eminence is a projection of the zygomatic bone, which is the most powerful portion of the midface.

To create a reconstructive algorithm, several classification systems have been proposed, mostly related to the extension, location, and tissue involvement of the defect. $^{2,3}$ One of the most useful and recently proposed classifications in terms of reconstruction is that of Cordeiro and Santamaria. ${ }^{3}$ The complex three-dimensional defect is classified under four types (types I to IV) as described in the following four subsections.

\section{TYPE I DEFECTS}

This type of defect, also known as limited maxillectomy, includes the resection of one or two walls of the maxilla, which is geometrically a hexahedron (a geometric structure with six walls). The anterior wall of the maxilla and the medial or orbital wall may also accompany type I defects. The palate is spared, however. In this type, the defect is localized centrally, and the paramedian lateral side of the midface is not affected. The outcome of the reconstruction is relatively better in type I defects because teeth, swallowing, and speech are not destroyed.
A

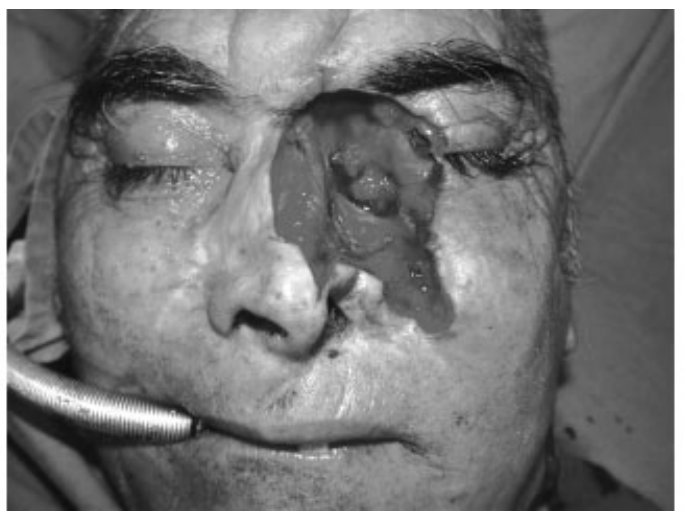

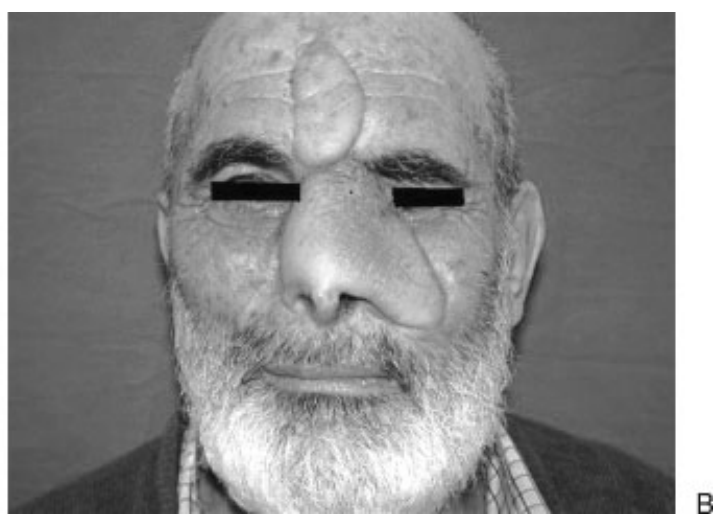

B

Figure 3 (A) Large nasal defect after wide resection of a recurrent basal cell carcinoma. (B) A dorsalis pedis flap was used to cover the defect. Postoperative view at 12 months. 


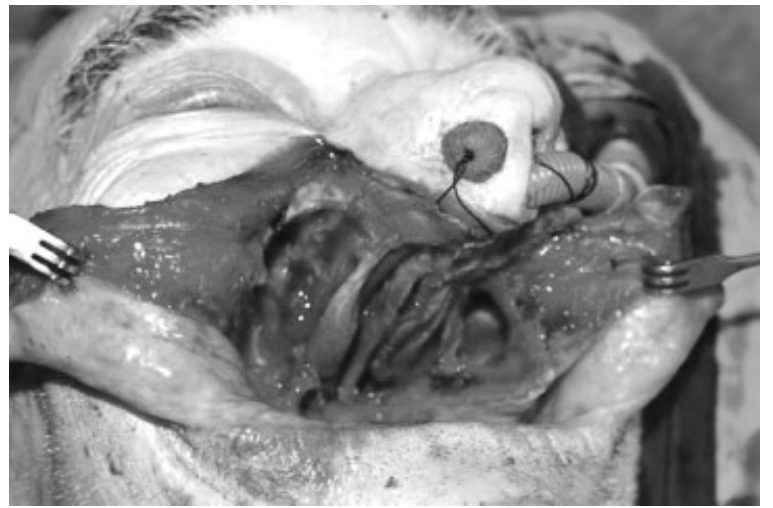

Figure 4 Intraoperative view of a subtotal maxillectomy case with resection of the lower part of the maxilla.

\section{TYPE II DEFECTS}

This defect is also known as subtotal maxillectomy, meaning the resection of the lower part of the maxilla with preservation of the orbital floor (Fig. 4). In this type, the lateral, medial, posterior, and anterior walls of the maxilla are removed with the palate. Nevertheless, the orbital floor is preserved, and the deformity is localized at the lower part of the maxilla. In type II defects, swallowing, chewing, and speech are disturbed because the maxillary arch and the palate are involved.

\section{TYPE III DEFECTS}

All six walls of the maxilla are completely removed, including the orbital floor (total maxillectomy) (Fig. 5). Type III defects can be further divided into two subcategories:

- Type IIIa: Although the orbital floor is removed, the orbital contents are preserved.

- Type IIIb: The orbital contents are also resected in addition to total maxillectomy.
In type III defects, the deformity is more obvious because, together with other deformities, vision problems such as protrusion, diplopia, ectropion, or even total blindness are observed. Eye socket reconstruction is also necessary in type IIIb defects.

\section{TYPE IV DEFECTS}

In this type of defect, the upper portion of the maxilla is removed with the orbital contents (orbitomaxillectomy) (Fig. 6). The palate is spared, but the removal of the orbital contents results in serious problems.

\section{RECONSTRUCTION OF COMPLEX DEFECTS}

Essentially, the aim of reconstruction is to eliminate the dead space; to maintain such functions as speech, swallowing, and mastication; to re-create the skeletal support; and to achieve the best cosmetic result.

Most maxillary defects are compound defects involving both soft and hard tissue. These defects exhibit functional impairment that makes reconstruction a complex and challenging issue. Local and free flaps are preferable in the majority of cases. Reconstruction with autogenous tissue provides sufficient tissue, and functional restoration is possible. Small partial defects such as type I can be managed with local flaps and combined with other techniques such as bone grafts and soft tissue injections. The temporalis muscle or osteomuscle, forehead, scalp, Abbe, pectoralis major, trapezius, latissimus dorsi, deltopectoral, and sternocleidomastoid are the most useful local flaps for small uncomplicated defects. However, local flaps cannot provide sufficient reconstruction in large and complex defects. These local and regional options usually have limitations in terms of rotation arc and inset. They may also have viability problems when used in the maximal range. These flaps

A
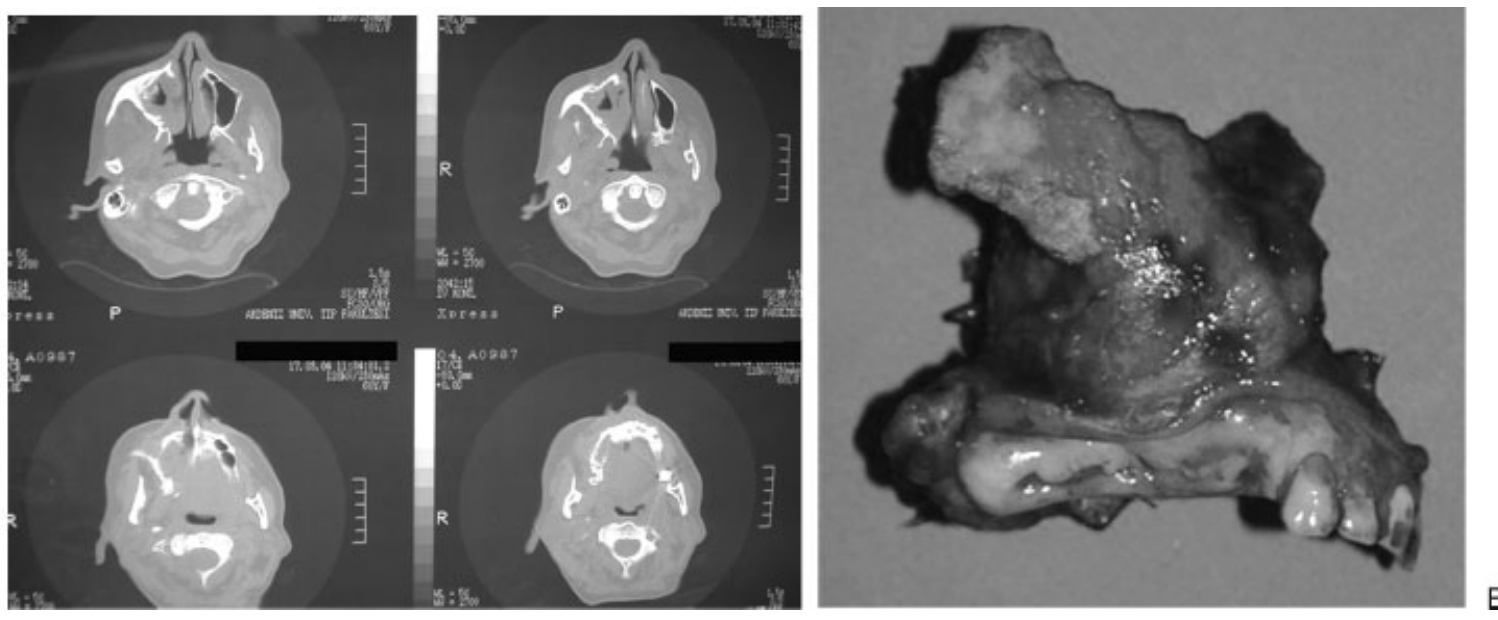

Figure 5 (A) A computed tomographic view of a patient with an adenocarcinoma originating from the maxillary sinus. (B) Resection material from total maxillectomy. 
A
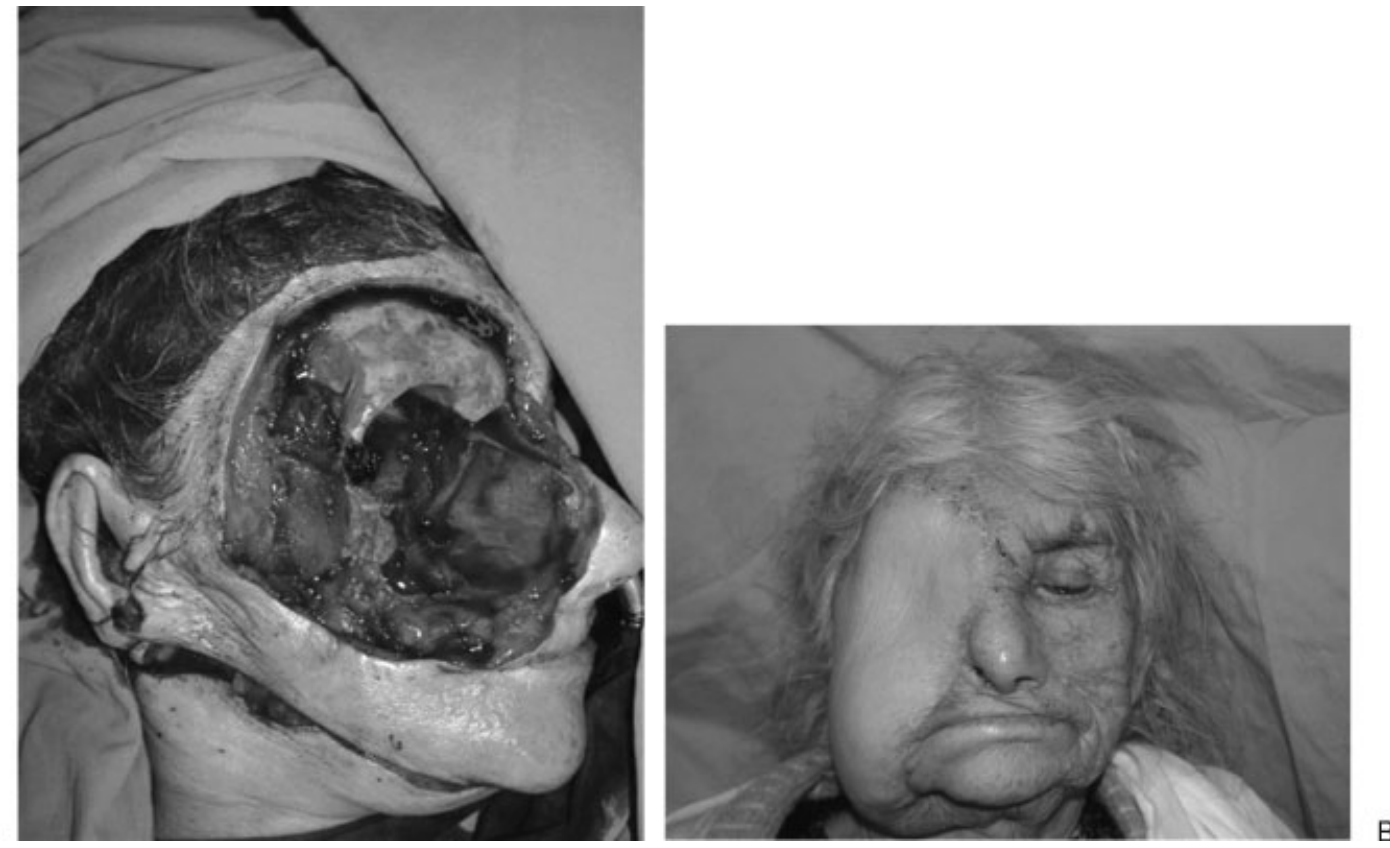

Figure 6 (A) Intraoperative view of an extensive resection of a squamous cell carcinoma resulting in orbitomaxillectomy defect (the upper portion of the maxilla was removed with the orbital contents). The defect was reconstructed with an ALT flap. (B) Postoperative view at 3 months.

can be considered when free flaps are contraindicated or have failed.

Free flaps are very useful for the reconstruction of midfacial defects, as in most head and neck reconstructions. Either single tissue or composite tissues can be transferred by microvascular reconstruction. The design and inset of the free flap offers great versatility, and functional reconstruction can be performed using free flaps (e.g., osseointegrated teeth to bone or functional muscle). Free flaps may also be combined with other techniques such as local flaps or grafts. The drawbacks of free tissue transfer are the need for a special technique and the long operating time.

Choosing the free flap for the best outcome requires special attention. If the defect involves either outside or inside resurfacing, only skin or mucosa defects for instance, then thinner flaps, such as the radial forearm, ulnar forearm, and lateral arm flaps or thinned ALT flap, are very useful. Nevertheless, the radial forearm is the best option because it is safe, reliable, and flexible. This flap can be combined with a small piece of radius for limited-size bone defects. It can also be combined with bone grafts. According to Cordeiro and Santamaria, the radial forearm flap is the flap of choice for type I and type II defects. ${ }^{3}$

Larger defects such as type III need bulkier flaps, and thicker flaps are necessary for reconstruction. The latissimus dorsi, scapular, and parascapular are safe alternatives, but they need position changes during surgery, which is not desirable. The flaps that can be harvested while the patient is in a supine position are the rectus abdominis, gracilis, and rectus femoris. Of these, the rectus abdominis is the most versatile option with its large skin island, long vascular pedicle, and safe vascularity. The skin island of the rectus abdominis musculocutaneous flap can be divided into multiple parts, so it is very useful for type III and type IV defects. For orbital support, bone grafts can be used, and a piece of rib can also be harvested with rectus abdominis flaps.

The recent popularity of perforator flaps has led to a major revolution in microvascular surgery. These flaps are very useful for the head and neck and have become the first choice when soft tissue is needed. ALT and latissimus dorsi (thoracodorsal artery) perforator flaps are good alternatives for the head and neck, but ALT seems better because there is no need for position changing, the vascular territory is safer, and donor site morbidity is minimal. All flaps used previously have been replaced by the ALT flap. Thin ALT flaps can be harvested in thin patients or the flap can be thinned primarily. Bulky flaps are also available, especially when the ALT flap is harvested as a musculocutaneous flap with the vastus lateralis muscle. Nowadays, the ALT flap is the primary choice in most centers dealing with head and neck reconstruction. ${ }^{4-7}$ ALT may be the first choice in every kind of defect for midface reconstruction. ${ }^{6,7}$ The drawbacks of perforator flaps are the need for meticulous, time-consuming dissection and for experience.

Another flap option for midface reconstruction is bone flaps. These may be osseous or osteocutaneous. The principal role of bone-containing flaps is to restore three basic structures: 
1. The maxillary arch.

2. The orbital floor.

3. The maxillary buttresses.

These flaps may sometimes be sufficient for successful reconstruction; however, the soft tissues of the bone flaps are limited, so combining these with other local or free flaps is mandatory. Flaps containing fibula, iliac crest, and rib, such as latissimus dorsi and rectus abdominis flaps, are the first choices for bone supply. Rib-containing flaps can supply enough tissue with bone, but the size and strength of the rib is limited, although useful for buttress reconstruction. ${ }^{8}$ The fibula is a good bone stock and has several advantages, such as having a large and pliable skin island, the availability of multiple osteotomies, and good donor site morbidity. The iliac crest also supplies a good bone reserve, although osteotomies cannot be performed easily, and the abdominal wall is weakened after iliac crest harvesting.

Another important point in maxillary arch reconstruction is the placement of the osteointegrated implant. This approach is very important for functional restoration, and the fibula is the best choice for strength and thickness when osteointegrated teeth are concerned. Osteointegrated teeth permit masticatory function and improve the cosmetic results. The combination of bone flaps with implants is the gold standard for maxillary reconstruction, and transferring a bone flap is otherwise useless in terms of functional reconstruction. Osteointegrated teeth are superior to other kinds of prosthesis because there is usually a lack of soft tissue to support the prosthesis. Osteointegration can be performed primarily or secondarily at another stage. Primary or simultaneous insertion of osteointegrated implants reduces operative sessions and hastens the rehabilitation process. ${ }^{9}$

\section{Maxillary Prosthesis}

The maxillary prosthesis requires special experience and has to be constructed individually for midface defects. Alloplastic prostheses provide an obturatory function between the orbital, maxillary, and oral cavities, as well as external coverage. These days, midface prostheses are made from acrylic or other polymers. These can be removed, cleaned, and reinserted. These kinds of prostheses need sufficient tissue to be inserted and fixed. The advantages of this technique are the lack of any need for surgery, observation of the field for recurrence, custom-made reconstruction, and no donor site morbidity.

However, the prostheses comprise nonliving tissues and may cause discomfort for the patient. Pressure ulcers and infections may occur, especially after improper use and with poor hygiene. The functional results are not superior because they cannot usually provide a stable support for teeth placement and good seal against leakage between cavities. Although autogenous tissues are preferable, midface prostheses are useful for patients when long operations are contraindicated or for very old or debilitated patients.

\section{CONCLUSION}

The management of large midfacial defects is a challenge for reconstructive surgeons. Defects that include composite tissue loss require more complicated procedures and consideration of both functional and cosmetic outcomes. Although most superficial skin defects can be covered by various standard reconstructive modalities, with the advances in microsurgery, microsurgical free tissue transfers have become the reconstructive method of choice for most large composite defects of the midface. The method of reconstruction should be selected individually, bearing in mind the medical situation; the age and prognosis of the patient; the size, extension, and composition of the defect; and the availability of local or distant tissues.

\section{REFERENCES}

1. Muzaffar AR, Adams WP Jr, Hartog JM, Rohrich RJ, Byrd HS. Maxillary reconstruction: functional and aesthetic considerations. Plast Reconstr Surg 1999;104:2172-2183; quiz 2184

2. Wells MD, Luce EA. Reconstruction of midfacial defects after surgical resection of malignancies. Clin Plast Surg 1995; 22:79-89

3. Cordeiro PG, Santamaria E. A classification system and algorithm for reconstruction of maxillectomy and midfacial defects. Plast Reconstr Surg 2000;105:2331-2346; discussion 2347-2348

4. Wei FC, Jain V, Celik N, Chen HC, Chuang DC, Lin CH. Have we found an ideal soft-tissue flap? An experience with 672 anterolateral thigh flaps. Plast Reconstr Surg 2002;109:22192226; discussion 2227-2230

5. Ozkan O, Coşkunfirat OK, Ozgentaş HE. An ideal and versatile material for soft-tissue coverage: experiences with most modifications of the anterolateral thigh flap. J Reconstr Microsurg 2004;20:377-383

6. Coskunfirat OK, Wei FC, Huang WC, Cheng MH, Yang WG, Chang YM. Microvascular free tissue transfer for treatment of osteoradionecrosis of the maxilla. Plast Reconstr Surg 2005;115:54-60

7. Ozkan O, Mardini S, Chen HC, Cigna E, Tang WR, Liu YT. Repair of buccal defects with anterolateral thigh flaps. Microsurgery 2006;26:182-189

8. Yamamoto $Y$, Minakawa $H$, Kawashima K, Furukawa $H$, Sugihara T, Nohira K. Role of buttress reconstruction in zygomaticomaxillary skeletal defects. Plast Reconstr Surg 1998; 101:943-950

9. Chang YM, Coskunfirat OK, Wei FC, Tsai CY, Lin HN. Maxillary reconstruction with a fibula osteoseptocutaneous free flap and simultaneous insertion of osseointegrated dental implants. Plast Reconstr Surg 2004;113:1140-1145 